\title{
28 Research Square \\ Quality Control of Mitochondria in Parkinson's Disease (PD) Using ATP And Bradford Assays
}

Al-Baraa Akram ( $\sim$ albraaakram94@gmail.com )

De Montfort University

Research Article

Keywords: heterogeneous, multifactorial, dopaminergic, pathogenesis, mitochondrial

Posted Date: January 3rd, 2022

DOI: https://doi.org/10.21203/rs.3.rs-1160696/v1

License: (c) (i) This work is licensed under a Creative Commons Attribution 4.0 International License.

Read Full License 


\section{Abstract}

Parkinson's disease is a heterogeneous, multifactorial and often complex disease characterized by motor impairment due to the presence of Lewy bodies and prominent degeneration of dopaminergic neurons in the substantia nigra.

Although the specific pathogenesis involving PD remains under investigation, mitochondrial dysfunction has been widely accepted as one of the major pathogenic pathways underlying the development of PD. Based on the hypothesis that depiction of $\mathrm{HtrA} 2$ (serine protease gene, mitochondrial precursor) might contribute to an increase in mitochondrial stress and transcriptional upregulation of the nuclear stressresponse CHOP gene. The present study aimed to analyze through laboratory-based research the role of HtrA2 and CHOP in the transmission of stress signaling and the consequent activation of mitochondrial quality control in Parkinson's disease using ATP and Bradford assays.

\section{Introduction}

\section{Definition:-}

Parkinsonism is a progressive neurological disorder of muscle movement characterized by any combination of tremor, rigidity, bradykinesia and progressive postural instability. Cognitive impairment is sometimes prominent.

\section{Causes:-}

The cause of the disease is unknown for most patients. The disease (especially idiopathic type) may be due to:

1. Destruction of dopaminergic neurons in the substantia nigra which is a part of the extrapyramidal system with a consequent reduction in dopamine action in the corpus striatum of the basal ganglia responsible for motor control

2. Overproduction and/or overactivity of acetylcholine triggers abnormal signaling, leading to loss of control of muscle movements.

\section{Drug therapy of parkinsonism:}

The central imbalance between the dopaminergic system (reduced dopamine activity) and cholinergic system (increased acetylcholine activity) is the backbone of designing drug therapy for the disease.

\section{Amantadine}

It is an influenza antiviral agent; it has an indefinite mechanism as an antiparkinsonian drug. Its mechanism of action is not definitely known, but it may work by:-

1- Increase dopamine release 
2-Inhibit cholinergic system

3- Blockade of NMDA excitatory receptors.

It may be used in mild cases of the disease. It is less effective than levodopa but more effective than anticholinergic drugs. Tolerance develops to its effect after 6-8 months of continued treatment.

Its side effects include anorexia, nausea, constipation, postural hypotension, restlessness and confusion.

\section{Mitochondrial structure and function:}

As shown in figure 1, mitochondria are highly regulated membrane-bound organelles involved in fundamental cellular and metabolic functions within eukaryotic cells, such as energy supply (ATP production) through oxidative phosphorylation (OXPHOS), calcium and metabolite supply, inflammation, intracellular signaling, cell proliferation and apoptosis (1).

The function and survival of dopaminergic neurons strongly rely on appropriate mitochondrial homeostasis. Mitochondrial dysfunction has been widely accepted as one of the major pathogenic pathways underlying the development of PD (2).

Parkinson's disease (PD) is the second most prevalent age related neurodegenerative disorder of the central nervous system (CNS), affecting over $2 \%$ of the worldwide population older than 65 years.

The neuropathology of PD is characterized by the presence of Lewy bodies and prominent degeneration of dopaminergic neurons in the substantia nigra pars compacta (SNpc), which leads to severe striatal dopamine (DA) deficits, resulting in motor impairment. Bradykinesia, resting tremors, slowness of movement, muscular rigidity and postural instability comprise some of the clinical motor features of PD. Patients may also develop nonclinical manifestations such as autonomic impairment, cognitive variations, depression, sleep disturbances and late stage dementia.

Changes in mitochondrial structure and dynamics, dysregulation of transcription factors, impairment and aggregation of protein components due to nuclear DNA and mitochondrial DNA (mtDNA) gene mutations or environmental stress are important causes associated with mitochondrial dysfunction (3). Accordingly, mutations in a broad range of primary genes have been implicated in mitochondrial dysfunction and are known to play a role in autosomal dominant and recessive forms of PD, such as u-Synuclein (SNCA, PARK1), Parkin (PARK2), Phosphatase and tensin homolog (PTEN)-induced putative kinase I (PINK/, PARK6), Leucine-rich repeat kinase 2 (LRRK2) and DJ-/(PARK7) (4).

Dysfunctions at mitochondria are removed via lysosomal degradation through the process of organellar quality control, so; called mitophagy as shown in figure 2. Mitophagy is triggered by numerous cellular events, including cellular stress and differentiation (5). This process is done by complexes of molecules that recognize dysfunction at mitochondria and target them for degradation through the autophagy 
or/mitophagy process done by ATG complexes importantly two of the genes that are mutated in PD, PINK/and Parkin are coordinators of the PINK I/Parkin-mediated mitophagy process (6).

Mice without function at $\mathrm{HtrA} 2 / 0$ mi protease activity showed induced upregulation of $\mathrm{CHOP}$, reduced mitochondrial function and premature aging in brain cells, due to an increase in mtDNA deletions (7).

\section{Materials And Methods}

\section{ATP Assay}

ATP levels were assessed using Cell Titer-Glo (Promega) following the manufacturer's instructions. Briefly, the genotypes were cultured in a 96-well plate, followed by drug treatment. For analysis, ATP levels were quantified using ATP standard curves using serial dilutions of ATP $(50 \mu \mathrm{L})$ solution in culture medium. Cells were incubated for 30 minutes. Chemiluminescence as shown in figure 3 , was measured using a SpectraMax (MS) spectrophotometer at a wavelength of $570 \mathrm{~nm}$. Furthermore, the ATP levels were normalized to the protein content, measured by the Bradford assay.

\section{Bradford Assay}

Protein levels were determined for normalization purposes using the Bradford assay. Briefly, the cells cultured in 96-well plates, as shown in figure 4, were washed with phosphate buffered saline (PBS) and incubated with Bradford solution (Sigma, Germany) for 30 minutes, followed by subsequent washing in PBS. In each experiment, samples were normalized to a standard curve prepared by a BSA stand and stock solution (in PBS) with serial dilutions made in the Bradford solution.

\section{Statistical Analysis}

Data were collected from biologics in triplicate for each genotype. All results are presented as the mean \pm SEM and were analyzed using one-way or two-way ANOVA.

\section{Results}

The different genotypes analyzed in this research were treated with $50 \mu \mathrm{M}$ of the neurotoxin 6-OHDA and $0.5 \mu \mathrm{M}$ of ADEP4 and ACP5. Then the cell viability was determined by MTT Assay. Cell viability showed a concentration-dependent reduction in the viability level of the different genotypes (Figure $5 \mathrm{~A}$ and $\mathrm{B}$ ). Compared to the vehicle control (DMSO), cell viability was mainly reduced in the $\mathrm{HtrA} 2 \mathrm{KO} / \mathrm{CHOP}$ genotype. Depletion of HtrA2 significantly increased the susceptibility to treatment with 6-OHDA $(p<$ 0.0001).

These results display a drug concentration-dependent effect on the cell viability of the genotypes, indicating that depletion of cellular content might be due to high concentrations of drug treatment with 6- 
OHDA. Therefore, the concentration of the drug will be proportional to the number of living cells as shown in figure (6).

\section{ATP production is decreased in HtrA2 KO/CHOP genotype:}

ATP production was determined via ATP assay nominalized by the measurement of protein levels using the Bradford method. Likewise, in the cell viability assay, the ATP assay also displayed a concentrationdependent reduction in energy production among the different genotypes and reflected both the cell viability and the metabolic status of the cells. The results illustrated that depletion of HtrA2 significantly increased susceptibility to drug treatment as shown in figure (7). Compared to the vehicle control (DMSO), the loss of energy production was significantly higher in the HtrA2 KO/CHOP genotype treated only with 6-OHD A $(p<0.0001)$.

\section{Discussion}

The HtrA2 gene comprises 4152 bases of DNA located in the forward strand of chromosome 2 and composed of eight exons. The gene encodes a $49 \mathrm{kDa}$ protein of 458 amino acid (aa) residues. The fulllength translated protein is located within the mitochondrial intermembrane space (IMS) and contains a trimeric pyramidal structure that goes through a multifaceted allosteric activation pathway and defines HtrA2 proteolytic regulation, specificity and activity. The protease comprises the Nterminal mitochondrial localization sequence (1-40 aa), a transmembrane domain (105-125 aa) that dictates the topology of the protein, a serine protease domain (150-343 aa) with catalytic triad residues (Ser306, His198 and Asp228), and one PDZ domain (364-445 aa) located at the C-terminal end (8).

$\mathrm{HtrA} 2$ proteases are able to activate or coordinate heterogeneous signaling pathways implicated in PQC. Following apoptotic stresses, the $\mathrm{N}$-terminus is cleaved, and the mature HtrA2 protein is released from the mitochondrial intermembrane space (IMS) into the cytosol. Numerous structural studies have presented an overall analysis of the molecular architecture of human HtrA2, although the accurate pathway by which the protein is activated remains unclear (9).

In mitochondrial biogenesis, $\mathrm{HtrA} 2$ predominantly operates as a chaperone, where it prevents polypeptide aggregation and assists with correct protein folding. Although movement of the PDZ domain, facilitated by stress conditions such as temperature increase or by PDZ-binding peptides, was suggested to trigger HtrA2 activity, which initiates its proteolytic function and degrades the impaired proteins, similar to the allosteric mechanism suggested for the regulation of its bacterial homolog (HtrA/DegP).

Genomic studies have demonstrated that displacement or HtrA2 proteolytic activity results in the accumulation of unfolded polypeptides within mitochondria, damage or mitochondrial respiration and elevated ROS yield, leading to impairment of mitochondrial bioenergetics (10). Additionally, depletion of the HtrA2 proteosome leads to transcriptional upregulalion or important nuclear stress-responses implicated in mitochondrial PQC mechanisms, including the transcription factor CHOP (CEBP homologous protein), which is differentially regulated upon depletion of this protease (11). 
CHOP/DDIT3 comprises 5150 bases of DNA located in the reverse strand region of chromosome 12 and composed of four exons. The gene encodes a $29 \mathrm{kDa}$ protein of 169 amino acid (aa) residues. The protease comprises two functional domains: an N-terminal domain sequence correlated with the transcriptional activation of the protein and a C-terminal basic leucine-zipper (bZIP) domain formed by a DNAbinding region of basic amino acids followed by a leucine zip per dimerization domain (12).

Bioinformatics research shows that the bZIP domains of the CHOP protease are highly conserved in humans and in different types of eukaryotic organisms. According to NCBI BLAST comparison, the mouse CHOP and human CHOP (Homo sapiens) protein sequences (obtained from the Protein Data Bank) have $88 \%$ identity and $91 \%$ similarity along their full length. A Cluslal Omega multiple sequence alignment performed with $\mathrm{CHOP}$ protein sequences from different species showed high sequence conservation in the bZIP domains of the analyzed species.

The bZIP domains present in CHOP are structurally similar in their DNA-binding and dimerization motifs.

Mitochondria coordinate ATP synthesis through the action of three interlinked processes: oxidative phosphorylation, tricarboxylic acid cycle (TCA) and fatty acid-oxidation (FAO) metabolism. Dysfunction of mitochondrial biogenesis and an increase in oxidative stress result in ATP depletion, which activates glycolysis. Augmentation of this glycolytic pathway can also be a consequence of environmental and genetic mutations (13)

Previous studies have indicated that loss of HtrA2 in mice decreased mitochondrial mass and potential, resulting in ATP depletion (14). The reduction in ATP synthesis might lead to UPR dysfunction, which is associated with an increase in oxidative stress and is likely to result in further impairment in mitochondria, leading to pathological mechanisms involved in neurodegeneration and apoptosis (15).

\section{Conclusions}

In conclusion, the current research supports the role of HtrA2 and CHOP in the transmission of stress signaling and the consequent activation of mitochondrial quality control in Parkinson's disease. Depletion of CHOP function impairs transcriptional activation of mitochondrial quality control signaling. Loss of HtrA2 results in significant upregulation of the molecular quality control marker $\mathrm{Hsp} 60$ and organellar quality control marker Atg5.

Drug treatment with 6-OHDA induced decreased cell viability and ATP product ions in the HtrA2 KO/CHOP genotype, displaying a drug concentration-dependent effect that reflects both the cell viability and the metabolic status of the cells. Drug treatment using 6-OHDA in conjunction with ADEP4 or ACP5 significantly improved cell survival and ATP production, indicating that these drugs might have a protective effect while recovering the effects of 6-OHDA. The findings provide new evidence that depletion of HtrA2 might contribute to an increase in mitochondrial stress and transcriptional upregulation of the nuclear stress response CHOP gene, both of which are involved in Parkinson's disease. 


\section{References}

1. Pickles, Vigie and Youle (2018) mitophagy and quality control mechanisms in mitochondrial maintenance, current biology Volume 28, Issue 4 page: 170

2. Luo et al. (2015) circuit architecture of VTA DA neurons revealed by systematic inputt]output mapping, Cell Volume 162, Issue 3 page 622

3. Kotiadis, Duchen and Osellame, (2014) mitochondrial quality control and communications with the nucleus are important in maintaining mitochondrial function and cell health, Biochimica et Biophysica Acta (BBA) - General Subjects, Volume 1840, Issue 4 page 1255

4. Cacabelos et al., (2017) parkinson's disease: from pathogenesis to pharmacogenomics, international journal of molecular sciences, volume 18, issue 3

5. Stephenson et al. (2009) identified of Atg5-dependent transcriptional changes and increases in mitochondrial mass in Atg5-deficient T lymphocytes, autophagy, volume 5 issue 5 page 625; Kaushik and Cuervo, (2015) humanin is an endogenous activator of chaperone-mediated autophagy, journal of cell biology, volume 217 page 635

6. Gumeni and Trougakos, (2016) cross talk of proteostasis and mitostasis in cellular homeodynamics, aging, and disease, oxidative medicine and cellular longevity, volume 2016 page 2-4

7. Moisoi et al., (2009) mitochondrial dysfunction triggered by loss of HtrA2 results in the activation of a brain-specific transcriptional stress response, cell death and differentiation, volume 16 page 450; Chen et al., (2014) the rice restorer if 4 for wild-abortive cytoplasmic male sterility encodes a mitochondrial-localized ppr protein that functions in reduction of wa352 transcripts, molecular plant volume 7 page 1497; Chakraborti and Dhalla, (2017) submitochondrial calpains in pathophysiological consequences, proteases in physiology and pathology page 385

8. Jarzab, Zurawa-Janicka and Lipinska, (2012) temperature-induced changes of HtrA2(Omi) protease activity and structure, Cell Stress and Chaperones, volume 51 page 36

9. Merski et al. (2017) molecular motion regulates the activity of the mitochondrial serine protease $\mathrm{HtrA2}$, cell death and disease, volume 8 page 2-10

10. Quiros and Langer (2015) new roles for mitochondrial proteases in health, aging and disease, nature reviews molecular cell biology, volume 16 page 345-359

11. Bose and Beal, (2016) mitochondrial dysfunction in parkinson's disease, journal of neurochemistry, volume139, IssueS1 Pages 216-231

12. Perez-Mancera and Sanchez-garcia, (2004) DDIT3 (DNA damage inducible transcript 3), Atlas of Genetics and Cytogenetics in Oncology and Hematology volume 8 page 242

13. Requejo-Aguilar and Bolanos, (2016) mitochondrial control of cell bioenergetics in parkinson's disease, free radical biology and medicine, volume 100 Page 123-137

14. Plun-Favreau et al, (2012) HtrA2 deficiency causes mitochondrial uncoupling through the $F_{1} F_{0}-A T P$ synthase and consequent ATP depletion, cell death and disease, volume 3 page 2 
15. Abou-Sleiman, Muqit and Wood, (2006) expanding insights of mitochondrial dysfunction in parkinson's disease, nature reviews neuroscience, volume 7 page 207

\section{Figures}

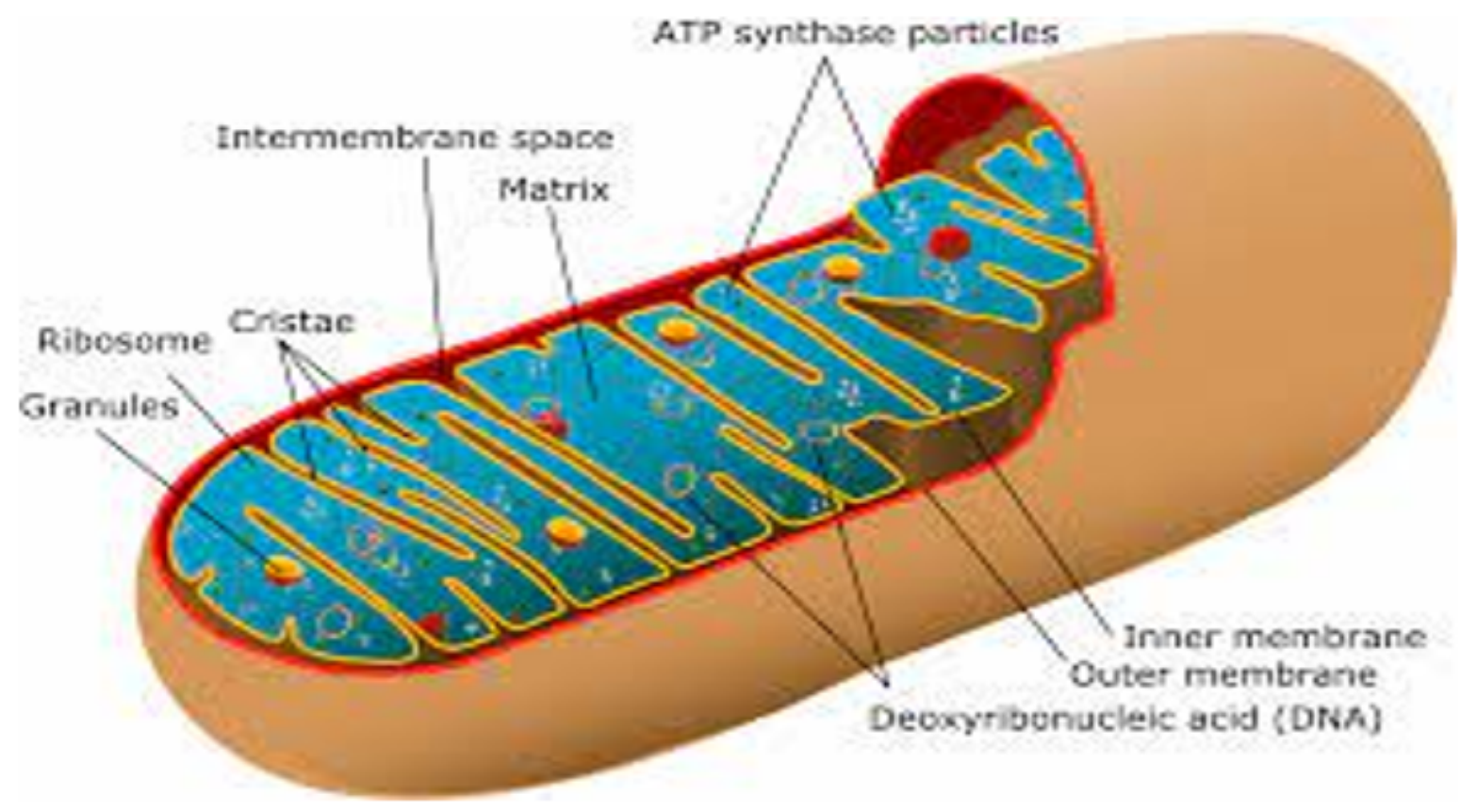

\section{Figure 1}

Illustration of the structure of mitochondria showing the outer and inner membranes, matrix, cristae, mitochondrial DNA and ribosomes 


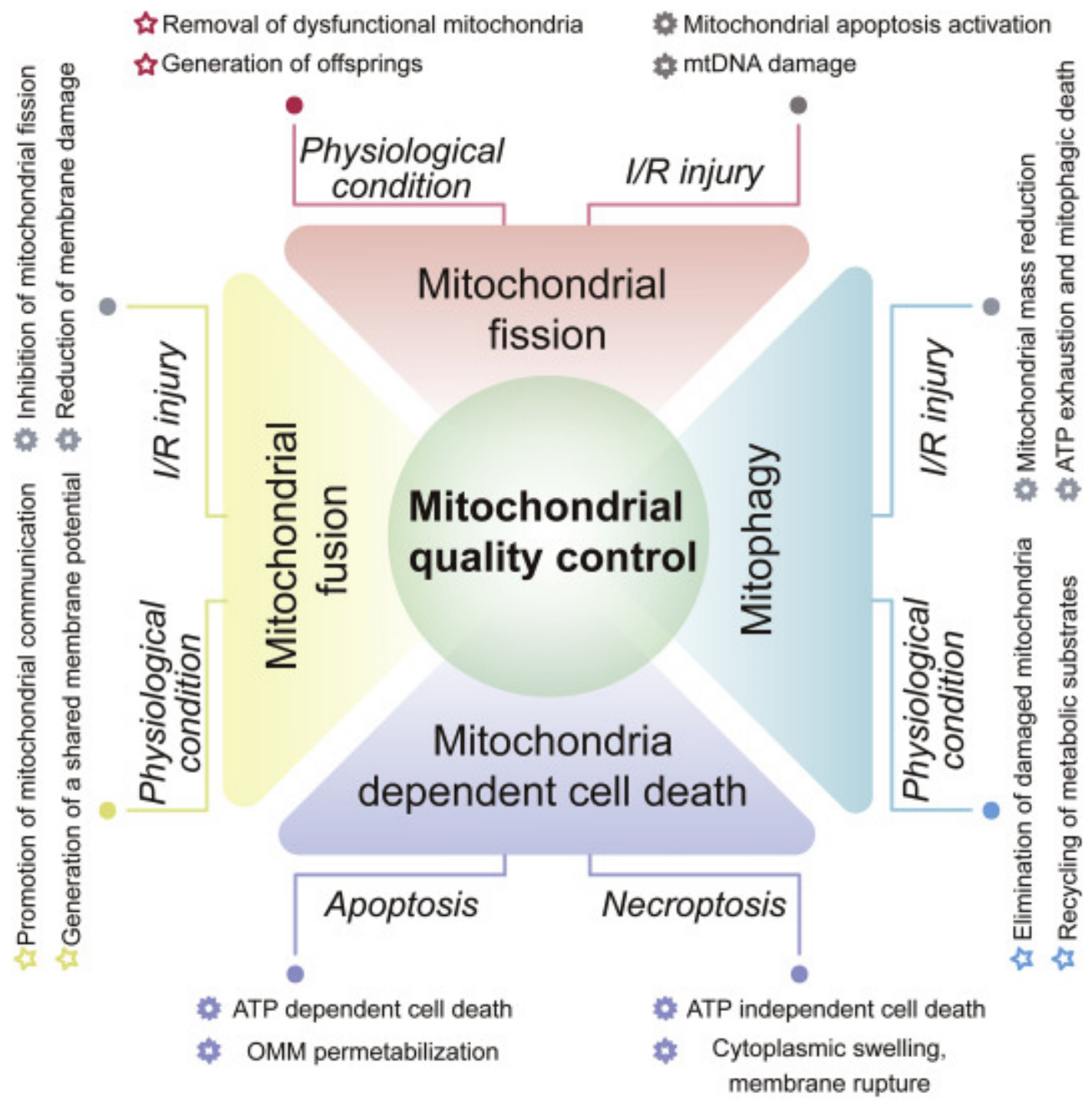

Figure 2

Mitochondrial quality control mechanisms as molecular targets in cardiac ischemia $\llbracket$ reperfusion injuryscience direct

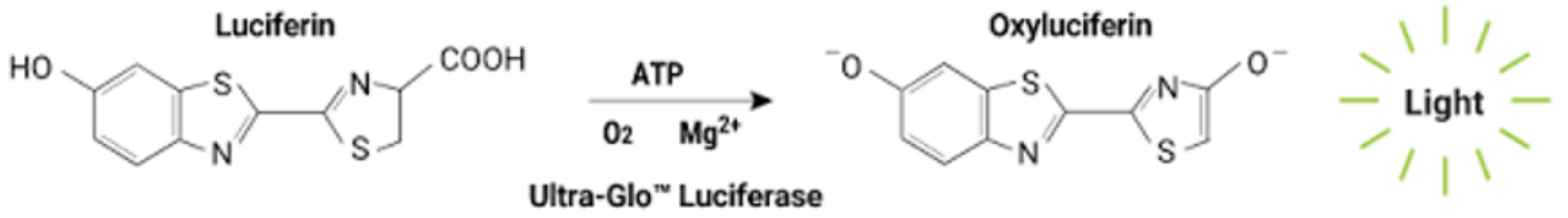

Figure 3

ATP assay illustrating the transformation of luciferin into oxyluciferin as an example of a chemiluminescence reaction 


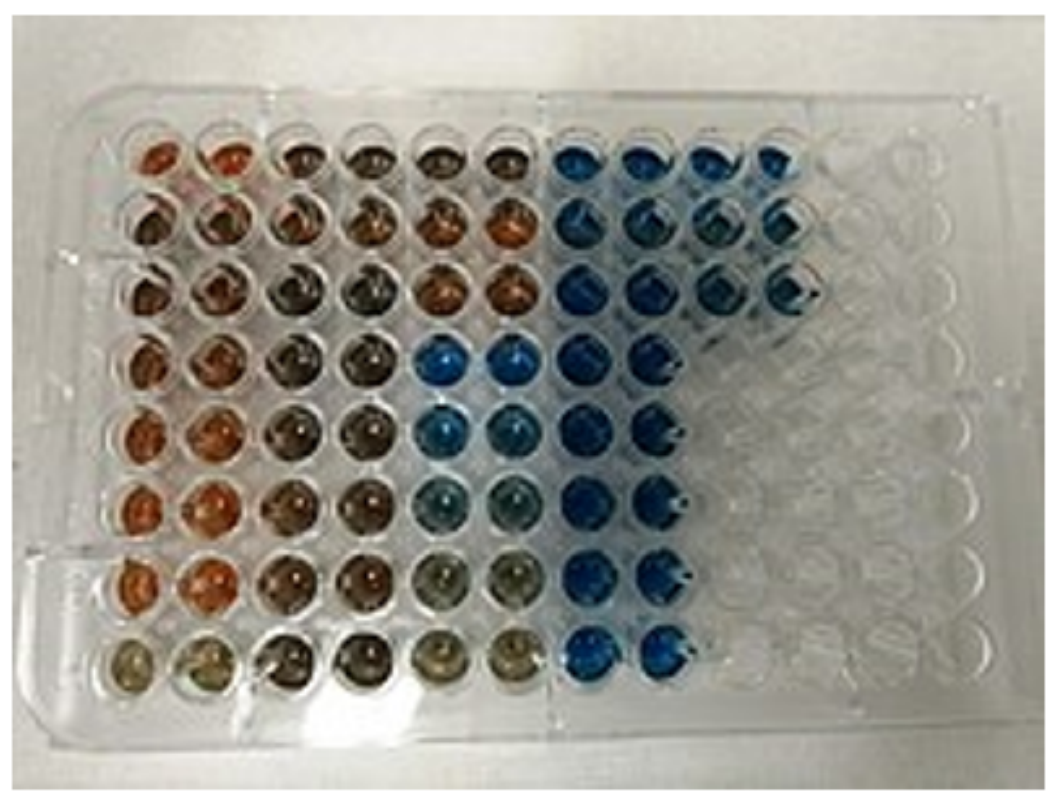

Figure 4

96-well plates of Bradford assay

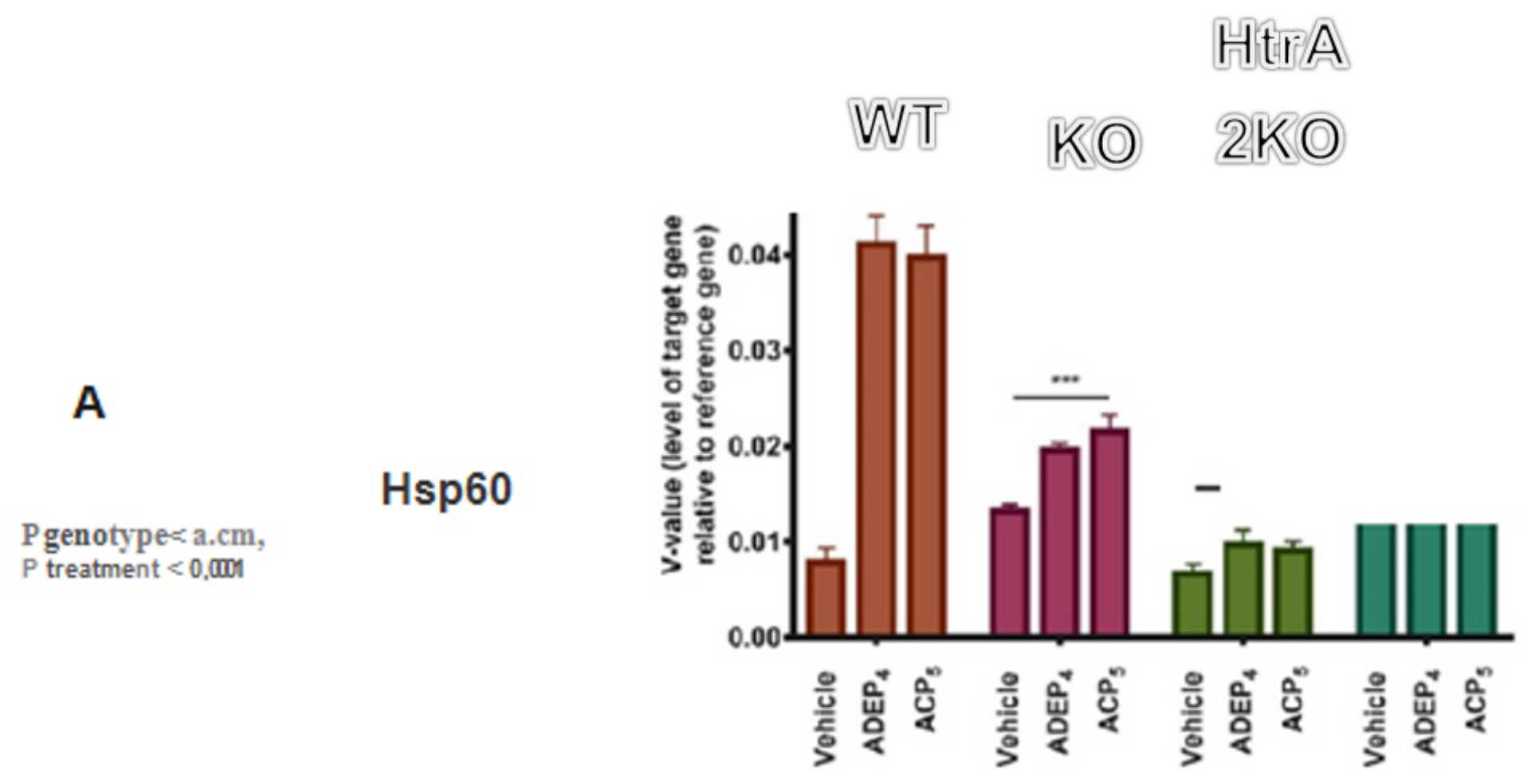

Figure 5

$\mathrm{CHOP}$ loss of function impairs transcriptional activation of mitochondrial quality control signaling. MEFs from wild-type (WT) (orange columns), CHOP-knockout (KO) (purple columns) and HtrA2 KO mice (green 
columns) were treated with ADEP4 and ACP5. RTALPCR analysis illustrated that depletion of HtrA2 results in significant upregulation of the molecular quality control marker (A) Hsp60 and organellar quality control marker (B) The data is represented as the mean \pm SEM. The statistical comparison was performed by two-way ANOVA followed by Tukey's multiple comparisons versus the vehicle control and between the indicated groups with $P<0.05 .{ }^{*} P<0.05, * * P<0.01 * * * P<0.001$
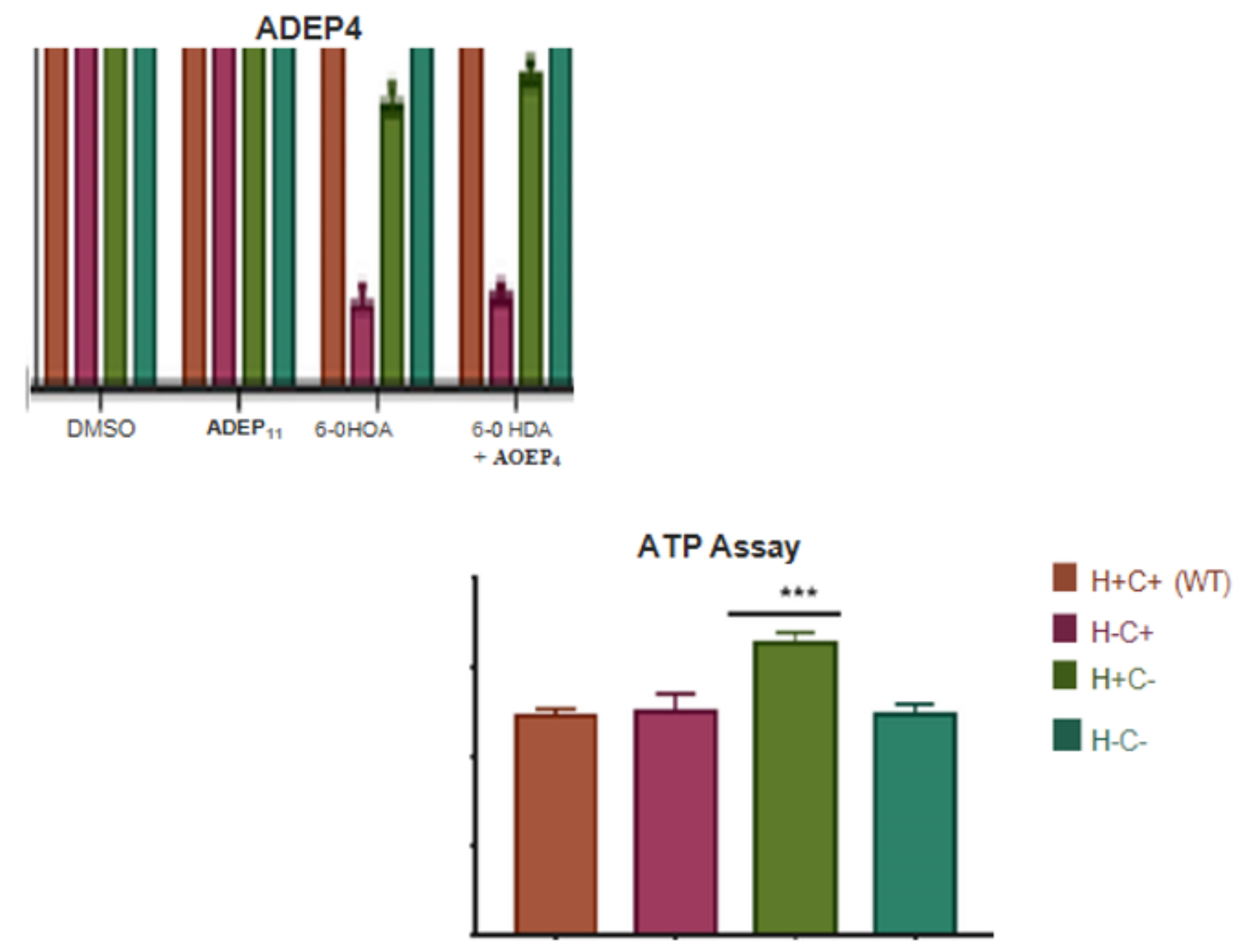

Figure 6

ATP production is decreased in the HtrA2 KO/CHOP genotype. Depletion of HtrA2 significantly increased the susceptibility to drug treatment Compared to vehicle control (DMSO), loss of energy production was significantly higher in $\mathrm{HtrA} 2 \mathrm{KO} / \mathrm{CHOP}$ genotype treated only with 6-OHDA. The energy level was significantly decreased in the HtrA2/CHOP KO genotype treated in conjunction with ADEP4 (A) or ACP5 (B). ATP product ions were significantly higher in the HtrA2/CHOP KO genotype (the data are presented as the mean \pm SEM). The statistical comparison was performed by one or two-way ANOVA 
followed by Bonferroni's multiple comparisons versus the vehicle control and between the indicated groups with $P<0.05$. $\cdot P<0.05$, "* $P<0.01$, "*"P $<0.001$

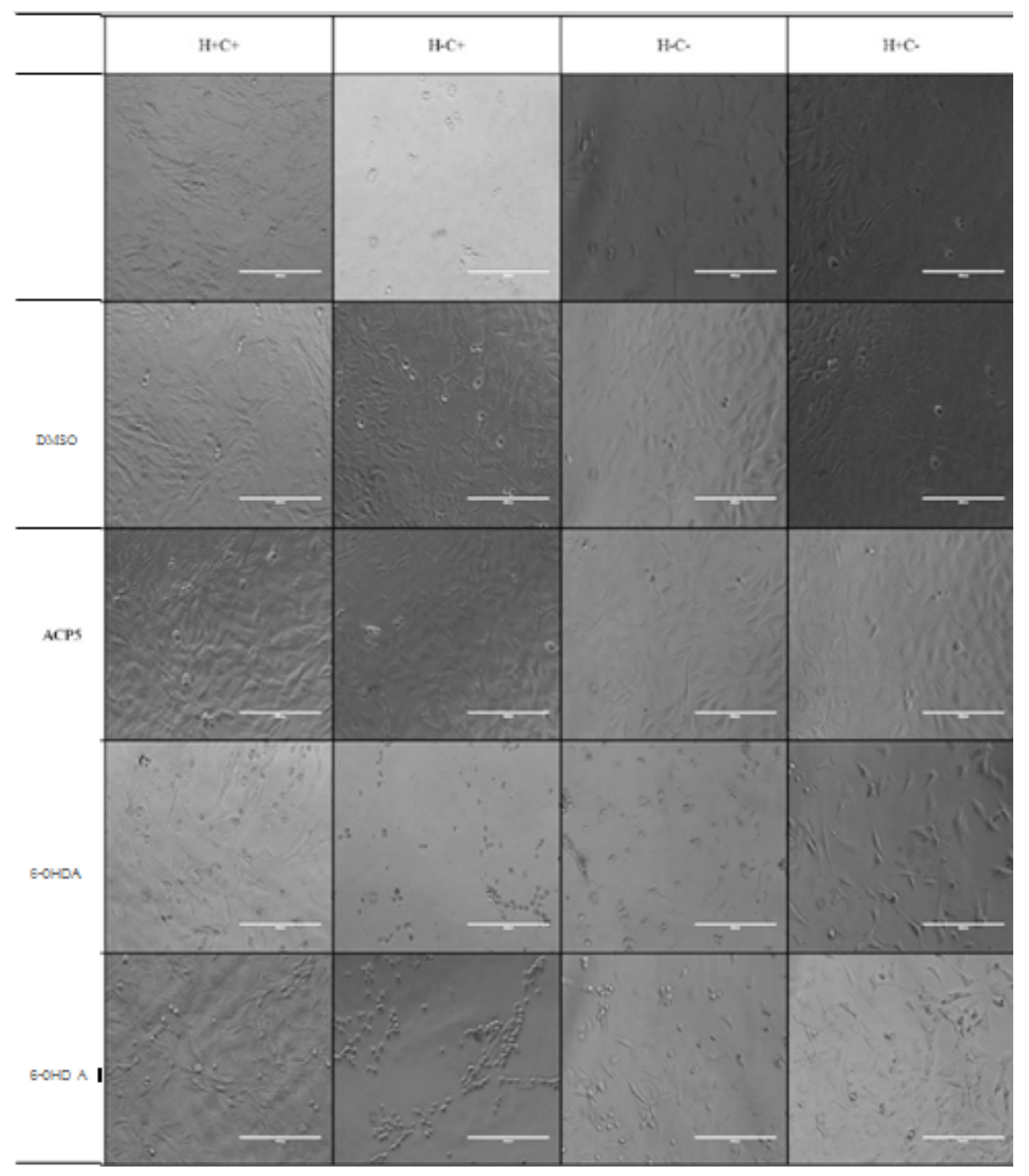

\section{Figure 7}

Microscopy visualization of mouse embryonic fibroblasts (MEFs) obtained from wild-type (WT), CHOP knockout (KO) and HtrA2 KO mice treated with 6-OHDA and ACP5. Compared to the other treatments, the $\mathrm{HtrA2/CHOP} \mathrm{KO}$ genotype had a significantly lower concentration of live cells than the well treated with only ACP5, which significantly improved cell survival. 